\title{
Tangence
}

\section{Les deux voix du roi}

Notes pour une nouvelle écoute de la parole souveraine de Henri III

\section{The two voices of the king}

Notes for newly listening to the sovereign speech of Henri III

\section{Pascal Bastien}

Numéro 93, été 2010

La majesté de la parole sous le règne de Henri III

URI : https://id.erudit.org/iderudit/045547ar

DOI : https://doi.org/10.7202/045547ar

Aller au sommaire du numéro

Éditeur(s)

Tangence

ISSN

1189-4563 (imprimé)

1710-0305 (numérique)

Découvrir la revue

Citer cet article

Bastien, P. (2010). Les deux voix du roi : notes pour une nouvelle écoute de la parole souveraine de Henri III. Tangence, (93), 9-16.

https://doi.org/10.7202/045547ar
Résumé de l'article

L'histoire de la voix a longtemps été considérée comme une entreprise impossible : fugitive par définition, elle serait un « faux bel objet d'histoire » en l'absence de sources pour l'écouter. La parole royale dispose cependant d'un certain nombre de sources et il devient possible d'en restituer les traces dans le cadre spatial et rituel où elle s'est exprimée. Une étude sur Henri III orateur ne peut faire l'économie d'une réflexion sur la matérialité de la parole, sur la théologie politique de la voix du roi, sur le caractère juridique de la voix souveraine et sur la position de cette parole dans l'espace où elle se fait entendre. 


\title{
Les deux voix du roi. Notes pour une nouvelle écoute de la parole souveraine de Henri III
}

\author{
Pascal Bastien, \\ Université du Québec à Montréal
}

\begin{abstract}
L'histoire de la voix a longtemps été considérée comme une entreprise impossible: fugitive par définition, elle serait un «faux bel objet d'histoire» en l'absence de sources pour l'écouter. La parole royale dispose cependant d'un certain nombre de sources et il devient possible d'en restituer les traces dans le cadre spatial et rituel où elle s'est exprimée. Une étude sur Henri III orateur ne peut faire l'économie d'une réflexion sur la matérialité de la parole, sur la théologie politique de la voix du roi, sur le caractère juridique de la voix souveraine et sur la position de cette parole dans l'espace où elle se fait entendre.
\end{abstract}

Il n'est plus nécessaire aujourd'hui d'insister sur la réputation de Henri III en tant que "prince orateur", que par ailleurs tous reconnaissaient déjà à l'époque: ennemis ou partisans du souverain étaient étonnés et charmés par l'élégance naturelle de sa parole et son attachement constant à l'apprentissage de la rhétorique. Nombre de travaux ont depuis insisté sur les dernières séances de l'Académie du Palais, sur les représentations d'orateur et les pratiques oratoires du roi, et sur sa formation en rhétorique $^{1}$ : cet article participe de cette réflexion et contribue à penser la résolution des conflits de cette période à la lumière de la puissance de la parole éloquente, idéal humaniste auquel tenait fort le dernier roi Valois.

1. Notamment, Robert J. Sealy, The Palace Academy of Henry III, Genève, Droz, coll. "Travaux d'Humanisme et Renaissance», 1981; et, plus récemment, Henri III, la rhétorique et l'Académie du Palais, dans Claude La Charité (dir.), Renaissance et Réforme/Renaissance and Reformation, vol. 31, $\mathrm{n}^{\circ} 4$, automne 2008. 
Cette attention portée à la rhétorique à l'époque des guerres de Religion amène l'historien qui est peu formé à cet art à s'intéresser à l'expérience de la "parole impérieuse», parole souveraine qui, au carrefour des mots et de la voix, doit aussi trouver ses lieux et ses temps pour être totalement efficace. L'art d'imposer sa volonté à autrui doit absolument être secondé par les conditions pratiques, spatiales et matérielles, pour qu'elle soit reçue et qu'on s'y conforme. Henri III ne fut certes pas le seul souverain à faire de sa parole l'un des instruments de sa politique; mais le souci qu'il avait de perfectionner "l'art de bien dire» permet sans doute d'aiguiser notre regard sur cette voix qu'on a alors plus volontiers capturée et commentée dans les textes. L'épisode est bien connu: après la harangue prononcée par Henri III aux États Généraux de 1576,

toute l'assemblée se leva pour le saluer, d'autant bonne affection que jamais Roy fut salué et honoré de ses sujets pour le grand contentement qu'ils avoient prins de l'ouyr si bien, disertement et élégamment parler, sans eslever ou baisser sa voix, qui estoit si bonne qu'il fut facilement entendu de tous les assistans, bien que la sale soit grande: sans interposer ou répéter une syllabe, sans hésiter ou se troubler aucunement ${ }^{2}$.

C'est la voix et le corps du souverain qui paraissent ici insuffler à la harangue, la compassion du souverain et la confiance des sujets qui l'écoutent. Objet d'histoire difficile à cerner, la voix est fugitive, évidemment inaccessible à l'oreille contemporaine. Pourtant, il ne suffit sans doute pas d'étudier le texte des harangues du roi pour en saisir toute l'efficacité, car ce sont aussi des rythmes, des intonations, voire des échos réverbérés dans l'espace d'une cour ou d'un théâtre ${ }^{3}$, auxquels on doit s'intéresser pour comprendre les singularités juridiques et politiques de la prise de parole royale. En s'intéressant aux harangues du roi, on risque d'orienter la lecture des textes vers une parole essentialisée, alors qu'il convient davantage de localiser et réunir les traces de parole susceptibles de s'inscrire dans une tradition et une liturgie.

Si le talent oratoire de Henri III fut salué par tous, déjà bien avant son accession au trône, le statut de sa parole et des modalités de son expression méritent, à mon avis, qu'on s'y penche davantage. Présentées sous forme de notes et d'hypothèses, ces réflexions

2. Recueil général des Estats tenus en France, Paris, au Palais, 1651, p. 234.

3. Voir le récent travail d'Arlette Farge, Essai pour une histoire des voix au XVIII siècle, Paris, Bayard, 2009. 
cherchent très modestement à proposer quelques voies d'enquête sur les «actes royaux de parole» qui, en soi et au-delà des formes rhétoriques de l'éloquence, faisaient de la voix du Roi «l'arc et la flèche» de la souveraineté ${ }^{4}$.

I. Bien qu'elle ne soit pas propre à Henri III, la parole souveraine doit absolument se distinguer de la parole $d u$ souverain. L'étude classique d'Ernst Kantorowicz sur Les deux corps du roi a analysé la construction juridique du Roi comme Loi animée et Justice vivante dont le corps intemporel aurait assuré l'immortalité et, partant, l'impossibilité d'une interruption du gouvernement et de la justice en raison des velléités de l'âge, de la maladie ou de la guerre $^{5}$. La théologie politique qu'il y explorait dévoilait non seulement le souverain comme incarnation terrestre et laïque du divin, mais réfléchissait aussi aux rituels de dédoublement des corps, comme ceux élaborés dans les obsèques royales jusqu'au $\mathrm{XVI}^{\mathrm{e}}$ siècle $^{6}$. La perpétuité du corps politique reposait sur cette fiction juridique. Si la réalité et l'efficacité politique et culturelle de cette construction a depuis été mise en doute par certains historiens ${ }^{7}$, il semble tout de même qu'au-delà du principe légal, la pratique rituelle et la culture politique du double corps aient intégré la façon d'imaginer et de concevoir la souveraineté dans la France d'Ancien Régime ${ }^{8}$. Or, il convient peut-être d'envisager le prolongement de cette théologie politique de l'incarnation du pouvoir dans la perspective d'une expérience de la voix ou, en d'autres termes, d'une matérialité de la parole. À la lecture des textes et des représentations figurées, il semble qu'il y ait eu non

4. Georges Dumézil, Apollon sonore et autres essais. Esquisses de mythologie, Paris, Gallimard, coll. «Bibliothèque des sciences humaines», 1982.

5. Ernst Kantorowicz, Les deux corps du roi. Essai sur la théologie politique au Moyen Âge [1957, éd. américaine], Paris, Gallimard, coll. "Bibliothèque des histoires ", 1989.

6. On peut lire, à ce propos, l'ouvrage de Ralph E. Giesey, Le roi ne meurt jamais. Les obsèques royales dans la France de la Renaissance, préface de François Furet, Paris, Flammarion, coll. "Nouvelle bibliothèque scientifique», 1987.

7. Par exemple, Alain Boureau, Le simple corps du roi. L'impossible sacralité des souverains français, $X V^{e}-X V I I I^{e}$ siècle, Paris, Éditions de Paris, coll. "Essais et Documents ", 2000; et "Ritualité politique et modernité monarchique», dans Neithard Bulst, Robert Descimon et Alain Guerreau (dir.), L'État ou le Roi. Les fondations de la modernité monarchique en France (XIVe-XVII siècles), Paris, Éditions de la Maison des Sciences de l'Homme, 1996, p. 9-25.

8. Voir notamment les nombreux travaux sur les représentations de Louis XIV, dont le maître essai de Louis Marin, Le portrait du roi, Paris, Minuit, coll. «Le sens commun », 1981. 
seulement deux corps, mais aussi deux voix du roi: une parole prononcée par le roi distincte de celle prononcée par le Roi, bien sûr en raison d'une mise en mots particulière, mais aussi d'une ritualisation conférant à l'expression du pouvoir une valeur de souveraineté.

II. La parole impérieuse, celle qui fait norme, qui fait la loi dans le lit de justice ou met hors la loi dans l'assassinat des Guise, doit tantôt être saisie dans son aspect de représentation, où le rôle de l'imaginaire est mis en avant, et tantôt dans l'acte social qu'elle constitue. Cette parole s'inscrit dans un système de liens, de rapports et de positions sociales strictement défini ${ }^{9}$. Rien ici qui soit très original, dans la mesure où la théorie des actes de parole a été défendue par Austin, Searle ou Bourdieu depuis déjà longtemps ${ }^{10}$. Comme le marteau du commissaire-priseur, le mot du roi arrête une discussion. En fait, le discours royal est un acte juridique: il ancre un dire dans une situation, produit — grâce à la bouche du locuteur - les conditions de son succès, fait de ses sujets des justiciables. Le discours du roi est un réel juridique, qui n'a pas à être soumis à l'épreuve de vérité. Le discours peut ne pas être accepté, mais alors celui qui n'entend pas la parole du roi est rebelle. Comme la loi, le roi construit ses propres événements et leurs enchaînements dans un espace plus large que la forme du discours ne le donne à voir.

III. À la différence du droit anglais, où le souverain fait appel à la loi, le roi de France, qui est fontaine de justice, n'a pas à évoquer le droit dans ses discours, puisqu'il est la loi. Il n'y a pas de sources extérieures à son pouvoir. Dans sa harangue de 1576, le roi affirmait devant tous les représentants du royaume sa conception de la souveraineté: l'autorité royale est transcendante, n'a pas à rendre compte de ses décisions, n'a que faire de l'opinion, puisque les actions du prince ne sont redevables qu'à Dieu ${ }^{11}$.

9. Voir, à ce propos, Fanny Cosandey (dir.), Dire et vivre l'ordre social en France sous l'Ancien Régime, Paris, Éditions de l'EHESS, coll. "Recherches d'histoire et de sciences sociales ", 2005.

10. John L. Austin, Quand dire c'est faire, Paris, Seuil, coll. «L'ordre philosophique", 1970; John R. Searle, Les actes de langage. Essai de philosophie du langage, Paris, Hermann, coll. "Savoir», 1972; Pierre Bourdieu, Ce que parler veut dire: l'économie des échanges linguistiques, Paris, Fayard, 1982.

11. Nicolas Le Roux, Un régicide au nom de Dieu. L'assassinat d'Henri III, ${ }^{\text {er }}$ août 1589, Paris, Gallimard, coll. «Les journées qui ont fait la France», 2006, p. 58 et suiv. 
IV. Henri III convoque deux fois l'assemblée des États, en plus de l'assemblée des notables de 1583. La fréquence des réunions révèle l'importance politique de l'institution; d'aucuns, il est vrai, affirment qu'elle témoigne surtout du peu d'efficacité de la politique royale. Pourtant, à Blois, les premiers États Généraux permirent le maintien de l'unité du royaume et l'édit de mai 1579, tandis que les seconds jurèrent solennellement l'édit d'Union de 1588.

Dès le XIII siècle, lorsque le roi voulait consulter ses sujets, il les convoquait par l'intermédiaire des communautés urbaines et rurales sous la forme et le rituel idéalisés des trois états de la société: les clercs, les nobles et le peuple. Il est important de restituer la parole du souverain dans l'organisation et le fonctionnement des assemblées appelées à la recevoir et à y répondre. Depuis les années 1950, l'histoire des assemblées représentatives et des États Généraux a considérablement avancé en dépassant la seule lecture des cahiers de doléances auxquels on les a longtemps réduits. En dépit des travaux novateurs du médiéviste Michel Hébert, l'historiographie a toujours l'habitude d'opposer une école "parlementariste» à une école «corporatiste»: la première insiste sur l'aspect constitutionnel de l'institution, tandis que la seconde privilégie l'analyse de la structure et de l'organisation sociale et corporatiste d'une société d'ordres. Les États Généraux

ont-ils une fonction constitutionnelle ou sont-ils de simples outils d'administration entre les mains des souverains? L'initiative en revient-elle aux sujets, réunis autour de la question de la défense de leurs privilèges, comme l'affirmait les corporatistes, ou appartient-elle au souverain? Enfin la question de la fiscalité et du consentement à l'impôt est-elle le pivot de cette histoire, comme tous l'entendent implicitement, ou faut-il tenir compte d'éléments complémentaires, plus difficiles à saisir et à mesurer, ainsi la fonction liturgique et cérémonielle qui est parfois dévolue à ces assemblées ${ }^{12}$ ?

Les États Généraux se font le lieu d'une médiation entre le souverain et ses sujets. Si nous disposons aujourd'hui d'une mémoire de papier, dans la publication de certaines harangues, doléances et procès-verbaux du déroulement des sessions de Blois, c'est en fait le jeu de la parole impérieuse dans le cadre du rituel des États

12. Michel Hébert, «Les assemblées représentatives et la genèse de l'État moderne en Provence (XIII-XV ${ }^{\mathrm{e}}$ siècle)", Genèse de l'État moderne en Méditerranée. Approches historiques et anthropologiques des pratiques et des représentations, Rome, École française de Rome, 1993, p. 269. 
Généraux, qui fonde toute la cohérence de l'événement et, à travers lui, qui contribue constamment à une réinvention de l'État royal. La description particulièrement détaillée de l'ordre et des gestes des participants à l'ouverture des États de 1576 et 1588 insiste suffisamment sur le chemin que doivent parcourir les paroles des acteurs. Les prises et les échanges de paroles tels qu'ils apparaissent dans les procès-verbaux et autres témoignages sur Henri III révèlent une "verticalisation" de la voix royale. La parole horizontale de la négociation se déplace. Le roi n'est plus éloigné mais élevé, tout de hauteur et de grandeur; le pouvoir semble s'être vraiment installé au firmament et c'est de ce ciel que procède un rapport compassionnel construit comme rapport politique: ce rapport compassionnel définit un "plus haut» très reconnaissable, puisque c'est le siège même du pouvoir; mais il est aussi de sa nature de déterminer un «plus bas», de désigner, parmi les sujets du prince, ceux qui sont les plus dignes de sa compassion et d'en faire par là un objet privilégié de l'action politique. La compassion cherche à favoriser l'effacement ou l'émiettement des autres passions politiques. Chaque fois qu'elle trouve à s'exprimer par la parole ou par les larmes, elle cherche à recréer un moment de cohésion unanime. "D'une bonne grace, parole ferme, haute et diserte», Henri III prononça sa harangue aux États Généraux de Blois de 1576. Jacques Amyot insistera évidemment, dans son Projet de l'éloquence royale, sur l'actio, c'est-à-dire la performance oratoire et corporelle, «la bonne grace de la voix, geste et contenance». Fondamentale, la "bonté de la voix", écrit-il, doit être haute, pleine, ferme tout en restant douce ${ }^{13}$. Miroir de l'âme, le visage royal peut assouplir et réconforter; les larmes du roi peuvent infléchir les opposants et les convaincre de la compassion du souverain ${ }^{14}$. La compassion est un renoncement de l'action politique ${ }^{15}$.

V. Le rôle de la parole royale impérieuse n'est plus celle du serment ou de l'échange de serments. Instrument du pouvoir monarchique aux côtés d'autres formes de communication politique avec lesquelles elle interagit, comme l'historienne Michèle

13. Jacques Amyot, Projet d'éloquence royale, préface de Philippe-Joseph Salazar, Paris, Les Belles Lettres, coll. "Le corps éloquent», 1992, p. 86.

14. Sur ce point, on peut lire Xavier Le Person, "Les larmes du roi": sur l'enregistrement de l'Édit de Nemours le 18 juillet 1585 », Histoire, économie et société, vol. 17, nº 3, 1998, p. 353-376.

15. Voir Robert Jacob, «Pietas ou la compassion. Langage de la loi et rhétorique des sentiments ", dans Pierre Ansart et Claudine Haroche (dir.), Les sentiments et le politique, Paris, L’Harmattan, coll. «Psychologie politique», 2007, p. 23-38. 
Fogel l'a bien montré dans Les cérémonies de l'information ${ }^{16}$, la parole du roi doit être comprise dans son rapport à Dieu. Dans le cadre où l'exercice même de l'autorité semble demander d'autant plus de contrôle que le rapport au divin confère à la parole d'un roi une vertu redoublée, le recours à la parole royale impérieuse présente aussi un caractère redoutable. Lorsque le roi prend la parole, il peut provoquer l'entrée dans le domaine de l'imprévisible, puisque chaque mot a valeur juridique. Les actes royaux de parole révèlent une tension entre le respect des règles et l'invention de nouvelles liées aux circonstances, et semblent constituer l'expression d'une légitimité en même temps qu'une forme de légitimation ${ }^{17}$. Les rituels de la parole font de la voix du souverain l'instrument tantôt du roi de guerre, tantôt du roi chrétien, tantôt du roi juge. C'est cette dernière qui sans doute confère le plus d'efficacité à la parole impérieuse. Dans les États Généraux comme dans le lit de justice, c'est d'ailleurs le chancelier qui est seul porteparole du roi ${ }^{18}$. Au carrefour de la sacralisation et de la banalisation, la voix du monarque prend sa force en reprenant et redistribuant les mots de ceux qui s'adressent à l'autorité. Ces paroles ne manquent jamais de renouveler la hiérarchie des acteurs ${ }^{19}$. Dans le cadre d'un conflit mais pas seulement, elle légitime, rend légal et perpétue une volonté politique. Dans les textes, on repérera des principes politiques d'ordre général, le plus souvent inscrits dans un modèle du passé dynastique qui fait référence et auquel le prince s'identifie, où est développée l'idée d'une monarchie juste, prudente et sage; alors que, dans le rituel, cette parole est tributaire de la position spatiale de l'énonciateur. Le mot doit tomber; il n'est pas une prière, il est une prescription. C'est parce que cette parole est légale que le discours ligueur se judiciarisa en interprétant la tyrannie dans un cadre politique et juridique plutôt que théologique et moral ${ }^{20}$. Il faut saisir, dans le déroulement des

16. Michèle Fogel, Les cérémonies de l'information dans la France du XVI au XVIII siècle, Paris, Fayard, coll. «Nouvelles études historiques», 1989.

17. Voir Stéphane Péquignot, «Les instructions aux ambassadeurs des rois d'Aragon (XIII ${ }^{\mathrm{e}}$-XIV ${ }^{\mathrm{e}}$ siècle). Jalons pour l'histoire de la fabrique d'une parole royale", Cahiers d'études hispaniques médiévales, $\mathrm{n}^{\circ} 31,2008$, p. 17-43.

18. Charles Figon, Discours des États et Offices tant du gouvernement que de la justice et des finances de France, Paris, Guillaume d'Avray, 1579.

19. Lire, à ce propos, François Foronda, «Du "dit au roi" au "dit royal". Traces et transformations de la parole du roi dans la Castille de la fin du $15^{\mathrm{e}}$ siècle", Hypothèses 2000, Paris, Publications de la Sorbonne, 2001, p. 231-259.

20. Arlette Jouanna, Le devoir de révolte. La noblesse et la gestation de l'État moderne, 1559-1661, Paris, Fayard, 1989. 
cérémonies, les véritables marqueurs de temps pour y voir la parole souveraine comme l'instrument d'activation de la vie politique. Cette parole ritualisée et publique est toujours offerte dans le cadre d'un jeu de confiance où la réconciliation, notamment dans l'organisation des États Généraux, est l'enjeu principal.

La parole devient une discipline dès le XIII ${ }^{e}$ siècle, avec le développement des universités, l'urbanisation, l'essor de la prédication, l'émergence et la diffusion de la procédure inquisitoire dans les cours criminelles du royaume, sans compter le renouveau du théâtre. Les miroirs des princes insistaient suffisamment sur l'importance pour les rois de bien maîtriser la parole ${ }^{21}$, les modèles de saint Louis et de Robert d'Anjou ${ }^{22}$ étant sollicités avec régularité. Alors que l'historiographie a l'habitude de penser la majesté par le silence qui en garde le mystère ${ }^{23}$, la parole du roi est pourtant fondamentale dans l'ordre et le mouvement de l'organisation sociale et monarchique ${ }^{24}$. La parole n'est pas qu'un souffle, elle obéit à des injonctions venues de plus haut. Henri III paraît avoir bien compris cette vertu presque magique de la voix et de la parole et a cherché à en faire usage et à en favoriser l'efficacité. Une compréhension étendue de la rhétorique royale ne peut se faire sans envisager la sacralisation de la parole royale, sa fonction et sa puissance juridique, sa position et sa circulation dans l'espace, ou le visage et les gestes qui lui donnent l'impulsion capable d'opérer le lien social entre les partis opposés, dans le moment passager d'une émotion partagée.

21. Ran Halévi (dir.), Le savoir du prince. Du Moyen Âge aux Lumières, Paris, Fayard, coll. «L'esprit de la cité», 2002.

22. Jacques Le Goff, Saint Louis, Paris, Gallimard, coll. «Bibliothèque des histoires", 1996; Darleen N. Pryds, The King Embodies the Word. Robert d'Anjou and the Politics of Preaching, Leyde, Brill, coll. «Studies in the history of Christian thought», 2000.

23. Ernst Kantorowicz, Les deux corps du roi, ouvr. cité; Jacques Chiffoleau, «Dire l'indicible. Remarques sur la catégorie du nefandum du XII ${ }^{e}$ au XV siècle", Annales ESC, no 45,1990 , p. 289-324.

24. Élodie Lecuppre-Desjardin, "Et le prince respondit de par sa bouche". Monarchal Speech Habits in Late Medieval Europe", dans Jeroen Deploige et Gita Deneckere (dir.), Mystifying the Monarch. Studies on Discourse, Power and History, Amsterdam, Amsterdam University Press, 2006, p. 55-64. 